\title{
Oral Microbiome and Response to Immunotherapy: Is It Time To Pay Attention?
}

\author{
Purushottam Lamichhane* \\ LECOM School of Dental Medicine, USA
}

*Corresponding author: Purushottam Lamichhane, LECOM School of Dental Medicine. 4800 Lakewood Ranch Blvd, Bradenton, FL 34211, USA.

Received Date: July 23, 2018

Published Date: August 06, 2018

\begin{abstract}
Microbiome studies have shown associations between the presence of certain bacteria in the gut and response to checkpoint inhibition (CPI) therapies in cancer patients. Studies looking at any such potential associations between oral microbiome and response to CPI therapies are lacking. Oral cavity is an important microbial habitat. Microbes present in the oral cavity influence not only the local and systemic immune homeostasis; but also the gastrointestinal microbial diversity. If associations exist between specific gut microbes and response to CPI therapies, it is reasonable to assume that such relationships exist between oral microbes and therapy response in cancer patients. Since the findings from such studies will have resounding implications on patient selection for therapies and prophylactic or therapeutic modulation of microbes for prevention or treatment of malignancies; it is crucial that dental and oral research community pays attention and joins this research effort to identify associations and elucidate mechanisms of oral microbial determinants of CPI therapy responses in cancer patients.
\end{abstract}

Keywords: Oral microbiome; Immunotherapy; Checkpoint inhibition; Resistance to therapy; Determinants of response; Biomarkers; Oral squamous cell carcinoma

Abbreviations: PD-1: Programmed Death Receptor 1; PD-L1: Programmed Death-Ligand 1; CTLA-4: Cytotoxic T-lymphocyteAssociated Protein 4; CPI: Checkpoint Inhibition; TME: Tumor Microenvironment; OSCC: Oral Squamous Cell Carcinoma; HNSCC: Head and Neck Squamous Cell Carcinoma

\section{Introduction}

The field of cancer therapies has benefitted immensely from the recent advances in immunotherapeutic approaches. The understanding of the role of checkpoint molecules, such as PD-1/ PD-L1, and CTLA-4 in mediating immunosuppression in the tumor microenvironment (TME) has led to development of antibodies that block these molecules and enhance anti-tumor immunity [1]. The success of these inhibitors has been highlighted by FDA approval of checkpoint blocking antibodies for use in patients with nonsmall-cell lung cancer, metastatic melanoma, classical Hodgkin's lymphoma, renal cell carcinoma, and metastatic or recurrent head and neck squamous cell carcinoma [2]. Despite this, majority of patients don't benefit from these treatments and identification of biomarkers of response has been slow. Studies have focused on understanding the innate barriers or the therapy induced adaptive resistances that hamper therapeutic response $[3,4]$. In line with that, susceptibility factors are being explored to inform the therapy response prediction and patient stratification. Among many, presence of specific microbes in the gut has been identified as potential determinant of therapeutic response to checkpoint inhibitor therapy in cancer patients [5-8]. What remains unexplored however is the contribution of specific microbes of the oral cavity in determination of success or failure of such therapies.

\section{Discussion}

High expression of PD-1/PD-L1 has been associated with poor outcomes in oral squamous cell carcinoma patients [9-11]. PD-1 blockade has been shown to prevent the development of carcinogen-induced oral premalignant legions and prevent the malignant transformation $[12,13]$. While clinical studies of PD-1 blockade exclusively on oral squamous cell carcinoma (OSCC) are not available, study on head and neck squamous cell carcinoma (HNSCC) has shown that a subset of patients benefit from this treatment; leading to the approval of PD-1 blocking antibody for 
patients with platinum-refractory recurrent and/or metastatic HNSCC $[14,15]$. These findings, however, further highlight that a majority of the patients do not respond to this therapy. Many recent studies have focused on identifying the determinants of response or failure to checkpoint blockade therapies. Such studies are lacking in the case of oral squamous cell carcinoma.

The field of microbiome research has gained momentum as of late, in part due to the studies showing associations between the presence of specific bacterial species in the gut and response to checkpoint blockade immunotherapy. One early study showed that presence of the commensal Bifidobacterium improved the efficacy of PD-L1 blockade therapy through a mechanism that depended on dendritic cell mediated priming of CD8+ T cells and their accumulation in the tumors [7]. Similarly, another study showed that anti-tumor activity of CTLA-4 blockade was enhanced by the presence of Bacteroides spp. (B. fragilis). This enhanced response was associated with presence of T-cells specific for these bacteria [8]. Melanoma patients that responded to PD-1 blockade immunotherapy were shown to have a presence of abundant Ruminococcaceae/Faecalibacterium in their gut [5]. Another study found that abundance of Akkermansia muciniphila in gut associated with response to PD-1 blockade in an IL-12 dependent manner [6]. The importance of gut microbiota in modulating therapy response was highlighted by the fact that consumption of antibiotics was associated with poor response to PD-1 blockade [6]. While the exact mechanism that underscores the efficacy of checkpoint inhibitors in presence of these bacterial species is unknown, heightened immune responses, including $\mathrm{T}$ cell infiltration into the tumors, secondary to the colonization of these bacterial species is thought to be the responsible factor [6-8].

Studies have demonstrated associations between presence of microbes and risk of squamous cell carcinomas [16-18]. Higher abundance of Corynebacterium and Kingella, which have the ability to metabolize carcinogens, has been shown to associate with decreased risk of HNSCC [16]. Porphyromonas Gingivalis and Fusobacterium nucleatum, however, were found to promote oral carcinogenesis in preclinical models [19].

Porphyromonas Gingivalis, Fusobacterium nucleatum and Actinomyces were shown to be increased in tumor sites of OSCC patients [20]. Fusobacterium were found to not only be enriched but also gradually increase in abundance with the progression of OSCC $[17,18]$. Despite these findings, whether the presence and abundance of these microbes is causative to OSCC or if their presence is rather facilitated by microenvironment changes with OSCC progression remains to be determined. Additionally, it is essential that contributions of other factors such as emotional stress of the patients, which play a crucial role in determining the composition of oral microbes as well as promoting the risk of periodontal diseases through behavioral and systemic changes [21], be considered while studying and establishing associations between oral microbes and risk of tumor development and progression. As such, psychological therapies may lend towards decreased risk of such microbial colonization and unveil whether true causal associations exist between these microbes and oral cancers development and progression.
Composition of oral microbiome has also been shown to associate with increased or decreased risk of developing pancreatic cancer. Specifically, presence of Porphyromonas gingivalis and Aggregatibacter actinomycetemcomitans associated with higher risk of pancreatic cancer while the presence of Fusobacteria and Leptotrichia associated with decreased risk of pancreatic cancer [22]. Similar studies are also available for other gastrointestinal cancer such as esophageal cancer [23]. While the associations of particular bacterial species with increased risk of cancers have been attributed to the persistent inflammation in response to these bacteria; if and how the presence of any microbial species confers resistance against development of malignancies is unknown. More importantly, whether presence of any specific oral microbe is associated with enhanced response to checkpoint blockade therapies remain to be determined.

\section{Conclusion}

Oral cavity is an extension of gastrointestinal system and represents a major habitat for microbes. Changes in microflora in the oral cavity affect both the local and systemic immune homeostasis and associations of oral microflora with oral and gastrointestinal cancers have been made. With recent studies showing correlations between presence of specific bacterial species in gut and response to checkpoint inhibitor therapies in various cancers, microbiome studies have started to focus on microbial biomarkers of response. Oral microbiome, on the other hand, has not yet been studied for the potential role of microbes on the success or failure of checkpoint inhibitor therapy response. It is essential that research studies be undertaken to detect relationship between oral microbes and response to checkpoint inhibitor therapies against oral and other cancers. Not only should such studies focus on identifying the oral microbial biomarkers or determinants of response to checkpoint blockade therapies, but also on elucidating the mechanisms inherent to the bacterial subspecies that regulate the outcome. Insights gained will not only allow for prophylactic and therapeutic modulation of microbes to prevent or treat oral cancers, but also inform rational combination of cancer therapies for successful clinical outcomes. Research on gut microbiome and association with immunotherapy responses has gained momentum. It is time for dentists and oral research community to pay attention and jump this research bandwagon.

\section{References}

1. Buchbinder EI, Desai A (2016) CTLA-4 and PD-1 Pathways: Similarities, Differences, and Implications of Their Inhibition. Am J Clin Oncol 39(1): 98-106.

2. Maleki Vareki S, Garrigos C, Duran I (2017) Biomarkers of response to PD-1/PD-L1 inhibition. Crit Rev Oncol Hematol 116: 116-124.

3. Jenkins RW, Barbie DA, Flaherty KT (2018) Mechanisms of resistance to immune checkpoint inhibitors. Br J Cancer 118(1): 9-16.

4. Lamichhane P, Karyampudi L, Shreeder B, Krempski J, Bahr D, et al. (2017) IL10 Release upon PD-1 Blockade Sustains Immunosuppression in Ovarian Cancer. Cancer Res 77(23): 6667-6678.

5. Gopalakrishnan V, Spencer CN, Nezi L, Reuben A, Andrews MC, et al. (2018) Gut microbiome modulates response to anti-PD-1 immunotherapy in melanoma patients. Science 359(6371): 97-103.

6. Routy B, Le Chatelier E, Derosa L, Duong CPM, Alou MT, et al. (2018) Gut microbiome influences efficacy of PD-1-based immunotherapy against epithelial tumors. Science 359(6371): 91-97. 
7. Sivan A, Corrales L, Hubert N, Williams JB, Aquino-Michaels K, et al. (2015) Commensal Bifidobacterium promotes antitumor immunity and facilitates anti-PD-L1 efficacy. Science 350(6264): 1084-1089.

8. Vetizou M, Pitt JM, Daillere R, Lepage P, Waldschmitt N, et al. (2015) Anticancer immunotherapy by CTLA-4 blockade relies on the gut microbiota. Science 350(6264): 1079-1084.

9. Maruse Y, Kawano S, Jinno T, Matsubara R, Goto Y, et al. (2018) Significant association of increased PD-L1 and PD-1 expression with nodal metastasis and a poor prognosis in oral squamous cell carcinoma. Int J Oral Maxillofac Surg 47(7): 836-845.

10. Mattox AK, Lee J, Westra WH, Pierce RH, Ghossein R, et al. (2017) PD-1 Expression in Head and Neck Squamous Cell Carcinomas Derives Primarily from Functionally Anergic CD4+ TILs in the Presence of PD-L1 TAMs. Cancer Res 77(22): 6365-6374.

11. Lin YM, Sung WW, Hsieh MJ, Tsai SC, Lai HW, et al. (2015) High PDL1 Expression Correlates with Metastasis and Poor Prognosis in Oral Squamous Cell Carcinoma. PLoS One 10(11): e0142656.

12. Wang J, Xie T, Wang B, William WN Jr, Heymach JV, et al. (2017) PD-1 Blockade Prevents the Development and Progression of CarcinogenInduced Oral Premalignant Lesions. Cancer Prev Res (Phila) 10(12): 684-693.

13. Chen Y, Li Q Ma D, Fang J, Luo L, et al. (2017) Blockade of PD-1 effectively inhibits in vivo malignant transformation of oral mucosa. Oncoimmunology 7(2): e1388484.

14. Economopoulou P, Kotsantis I, Psyrri A (2016) The promise of immunotherapy in head and neck squamous cell carcinoma: combinatorial immunotherapy approaches. ESMO Open 1(6): e000122.
15. Qi X, Jia B, Zhao X, Yu, D (2017) Advances in T-cell checkpoint immunotherapy for head and neck squamous cell carcinoma. Onco Targets Ther 10: 5745-5754

16. Hayes RB, Ahn J, Fan X, Peters BA, Ma Y, et al. (2018) Association of Oral Microbiome with Risk for Incident Head and Neck Squamous Cell Cancer. JAMA Oncol 4(3): 358-365.

17. Yang CY, Yeh YM, Yu HY, Chin CY, Hsu CW, et al. (2018) Oral Microbiota Community Dynamics Associated With Oral Squamous Cell Carcinoma Staging. Front Microbiol 9: 862.

18. Zhao H, Chu M, Huang Z, Yang X, Ran S, et al. (2017) Variations in oral microbiota associated with oral cancer. Sci Rep 7(1): 11773.

19. Perera M, Al-Hebshi NN, Speicher DJ, Perera I, Johnson NW (2016) Emerging role of bacteria in oral carcinogenesis: a review with special reference to perio-pathogenic bacteria. J Oral Microbiol 8: 32762.

20. Nagy KN, Sonkodi I, Szoke I, Nagy E, Newman HN (1998) The microflora associated with human oral carcinomas. Oral Oncol 34(4): 304-308.

21. Reners M, and Brecx M (2007) Stress and periodontal disease. Int J Dent Hyg 5(4): 199-204.

22. Fan X, Alekseyenko AV, Wu J, Peters BA, Jacobs EJ, et al. (2018) Human oral microbiome and prospective risk for pancreatic cancer: a populationbased nested case-control study. Gut 67(1): 120-127.

23. Le Bars P, Matamoros S, Montassier E, Le Vacon F, Potel G, et al. (2017) The oral cavity microbiota: between health, oral disease, and cancers of the aerodigestive tract. Can J Microbiol 63(6): 475-492. 